\title{
Use of radial symmetry for the calculation of cylindrical absorption coefficients and optimal capillary loadings
}

Authors

\author{
Peter Khalifah ${ }^{\mathrm{ab} *}$ \\ ${ }^{\text {a } D e p a r t m e n t ~ o f ~ C h e m i s t r y, ~ S t o n y ~ B r o o k ~ U n i v e r s i t y, ~} 100$ Nicolls Rd, Stony Brook, NY, 11794, USA \\ ${ }^{b}$ Department of Chemistry, Brookhaven National Laboratory, Upton, NY, 11973, USA
}

Correspondence email: kpete@bnl.gov

Synopsis Methods for numerically evaluating cylindrical absorption corrections which fully utilize radial symmetry to simplify distance calculations are presented. An analysis of the resulting data suggests that the best quality transmission diffraction data will generally be obtained when strongly absorbing samples are diluted by a factor of $F=(4 / 3) \mu \mathrm{R}$ in a maximal radius capillary.

Abstract The problem of numerically evaluating absorption correction factors for cylindrical samples has been revisited using a treatment that fully takes advantage of the sample symmetry. It is shown that the path lengths for all points within the sample at all possible diffraction angles can be trivially determined once the angle-dependent distance distribution for a single line of points is calculated. This provides advantages in both computational efficiency and in gaining an intuitive understanding of the effects of absorption on diffraction data. A matrix of absorption coefficients calculated for $\mu R$ products between 0 and 20 for diffraction angles $\theta_{\mathrm{D}}$ of $0^{\circ}$ to $90^{\circ}$ were used to examine the influence of (1) capillary diameter and of (2) sample density on the overall scattered intensity as a function of diffraction angle, where $\mu$ is the linear absorption coefficient for the sample and $R$ is the capillary radius. Based on this analysis, the optimal sample loading for a capillary experiment to maximize diffraction at angles of $0-50^{\circ}$ is in general expected to be achieved when the maximum radius capillary compatible with the beam is used, and when the sample density is adjusted to be $3 /(4 \mu R)$ of its original density.

\section{Introduction}

Powder diffraction is the primary analytical experimental tool for the study of crystalline materials. While the major role of powder diffraction has traditionally been phase identification through pattern matching, the use of powder diffraction data for structure refinement by Rietveld methods and even the use of powder diffraction data for $a b$ initio structure solution has become widespread in present times. This is due to both the advent of modern instrumentation (allowing the collection of very high quality diffraction data with narrow peak widths and good counting statistics 
over an extended range of $d$-spacings) and the development of improved computing tools (hardware, software, and methods) for data analysis. In all cases, the determination of crystallographic structures from diffraction data requires accurate knowledge about the amplitude of reflection structure factors, which are inferred from experimentally measured diffraction peak intensities.

Sample absorption has the potential to strongly modify observed diffraction peak intensities. If this absorption is not treated properly, it will result in incorrectly determined displacement parameters (Hewat, 1979), and potentially also give rise to incorrect site occupancies or phase fractions since these parameters are often closely correlated with displacement parameters. Since the determination of these crystallographic parameters is typically the central goal of powder diffraction structural studies, it is very important to appropriately correct for absorption effects in powder diffraction experiments. In the flat-plate Bragg-Bretano geometry that is routinely used in laboratory powder diffraction studies, absorption generally occurs in an angle-independent manner and can be safely neglected in most cases. However, many of the advanced diffraction beamlines at national facilities operate almost exclusively in a Debye-Scherer geometry, with samples typically loaded into a cylindrical capillary or can with a diameter ranging from $0.1-10 \mathrm{~mm}$, depending on the nature of the synchrotron or neutron beamline. For example, the 11-BM beamline at the Advanced Photon Source synchrotron utilizes a robotic autoloader for samples mounted inside $0.8 \mathrm{~mm}$ diameter plastic capillaries, allowing data to be collected on hundreds of samples yearly (Wang et al., 2008). Similarly, the POWGEN beamline (Huq et al., 2011) at the Spallation Neutron Source has a 24sample changer which allows for a large throughput of samples mounted in $6 \mathrm{~mm}$ or $8 \mathrm{~mm}$ diameter vanadium cans. Since data on these and comparable beamlines are collected in transmission mode, absorption effects can only rarely be safely neglected.

A cylindrical absorption correction is the appropriate method for treating sample absorption effects in the Debye-Scherer data collection geometry. A diffraction sample is assumed to be homogenous, and to therefore absorb X-rays according to the functional form of Equation 1.

$$
T=I / I_{0}=e^{-\mu \cdot L}
$$

The diffracted beam intensity $(I)$ is attenuated relative to the incident beam intensity $\left(I_{0}\right)$ in an exponential manner which is proportional to the total path length travelled through the sample $(L)$ with a proportionally constant of $\mu$ that is the linear absorption coefficient and which therefore has units of inverse distance. The ratio of $I / I_{0}$ is a transmission factor that can adopt values between zero and one and is here denoted by the variable $T$, though it should be noted in some classic work transmission has instead been denoted by the variable $A$ representing an attenuation factor (Dwiggins, 1972, 1975). Similarly, an absorption factor $A^{*}=1 / \mathrm{T}=I_{0} / I$ has also been classically used, and this variable has a value of one in the absence of absorption, and takes larger values when absorption is high. Although the exponential expression for absorption is simple, the overall absorption from a 
sample is generally complex to calculate since the different volume (V) elements within the sample are not constrained to have the same values of $L$. For each individual volume element, it is necessary to calculate the path lengths traversed by the incident beam $\left(L_{1}\right)$ and diffracted beam $\left(L_{2}\right)$, with $L=$ $L_{1}+L_{2}$. The absorption from the entire sample is thus represented by the volume integral given in Equation 2, where the parameters that depend on the diffraction angle $\left(\theta_{\mathrm{D}}\right)$ are indicated with a subscript $\theta$. This formula holds for any three-dimensional object, and is also relevant to absorption correction calculations for single crystal diffraction experiments.

$$
A_{\theta}=(1 / V) \int_{V} e^{-\mu \cdot L_{\theta}} d V
$$

In a conventional powder diffraction experiment where the scattering is measured only in a single plane of diffraction, the problem is reduced to a two-dimensional (2D) rather than a threedimensional (3D) one due to constant circular cross-section of the capillary. The volume integral can therefore be simplified to the area integral given in Equation 3, where the variable $A$ here denotes area. However, the path length to any area element will still depend on the angle of diffraction $\left(\theta_{\mathrm{D}}\right)$, and must be separately calculated for each diffraction angle over which data are collected (i.e., over a $2 \theta_{\mathrm{D}}$ range of $5-140^{\circ}$ ). Equation 3 is universally applicable to the problem of calculating cylindrical absorption coefficients when all scattering is in a single plane of diffraction.

$$
T_{\theta}=(1 / A) \int_{A} e^{-\mu \cdot L_{\theta}} d A
$$

Analytical expressions for cylindrical absorption have previously been derived. However, they suffer from the two limitations of (1) only describing two specific cases, namely diffraction angles $\theta$ of $0^{\circ}$ or $90^{\circ}$, and (2) having a sufficiently complex functional form involving modified Bessel and Struve functions such that numerical evaluation of their values is still required. Therefore, numerical methods provide the only practical method for obtaining precise values for $\mathrm{A}_{\theta}$ over a wide range of diffraction angles. Accurate tabulated transmission factors for selected diffraction angles and absorption coefficients have been available since the 1970s (Dwiggins, 1972, 1975, Hewat, 1979, Rouse et al., 1970), and more modern investigations have explored more complex geometries such as annular samples which may require the use of more than one adsorption coefficient (Bowden \& Ryan, 2010, Schmitt \& Ouladdiaf, 1998), as in the case of an adsorbing sample in an adsorbing vanadium can. However, the need to separately calculate $A_{\theta}$ by numerically integrating over many points across a fine $2 \mathrm{D}$ grid for each diffraction angle of interest and the need to recalculate $\mathrm{A}_{\theta}$ if the absorption coefficient $\mu$ is varied together make the direct calculation of absorption factors too slow too incorporate into modern software for Rietveld refinement. Software packages instead typically make use of simpler approximate functional forms such as that of Sabine (Sabine et al., 1998) or Lobanov and alte da Veiga (Lobanov \& alte da Veiga, 1998) in order to simply approximate adsorption effects. 
In the present work, the numerical calculation of cylindrical absorption corrections has been revisited with methods that fully take advantage of the radial symmetry of this problem. These methods allow an intuitive understanding of the influence of sample geometry on absorption to be obtained since all the information needed to calculate absorption coefficients can be obtained merely using a 1-dimensional line of points within a unit circle. This method leads to a concise and efficient algorithm for calculating overall sample absorption coefficients. Furthermore, absorption coefficients obtained in this manner are used to calculate optimal sample loadings for maximizing the diffraction intensity at selected diffraction angles. The influence of sample density on diffraction intensity is also quantitatively analysed in the context of dilution, and a widely applicable method for judging the optimal capillary sample loading procedure is proposed.

\section{Methods}

\subsection{Distance calculations using radial coordinates}

In the present calculations of cylindrical absorption coefficients, the numerical area integration of Eqn. 3 has been carried out in a grid defined by radial coordinates $(r, \phi)$ rather than Cartesian coordinates $(x, y)$, as illustrated in Figure 1. In all cases, it is necessary to calculate the total distance $(L)$ that X-rays will travel through the sample when they are diffracted from each area element with $L=L_{1}+L_{2}$, where $L_{1}$ is the distance traversed by the incident beam and $L_{2}$ is that traversed by the diffracted beam. The two distances $L_{1}$ and $L_{2}$ vary from point to point across these grids and for any given point will vary as the diffraction angle $\theta_{\mathrm{D}}$ is changed. This makes the calculation of absorption coefficients computationally intensive.
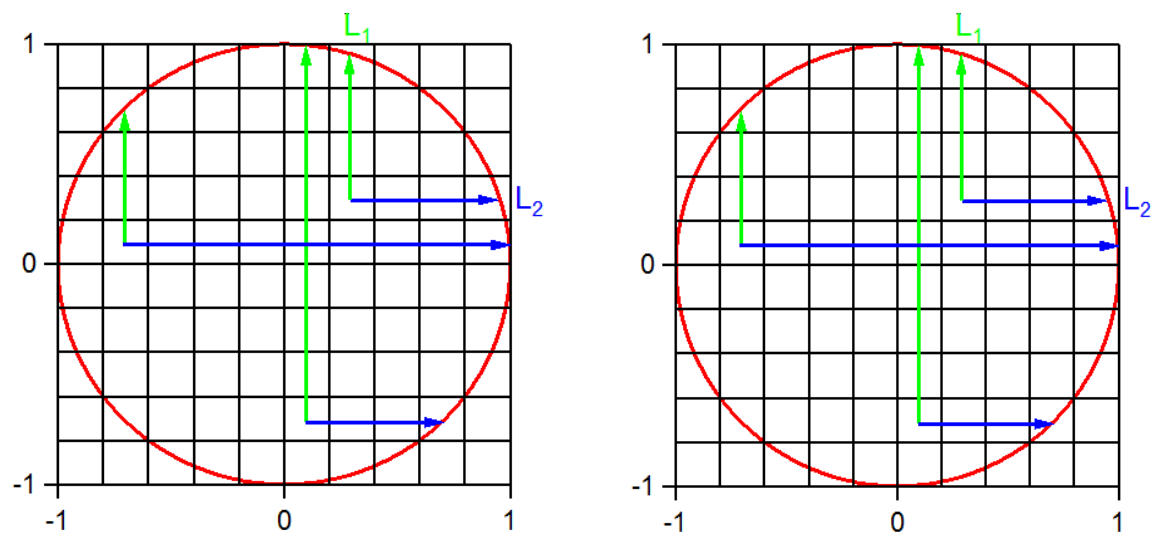

Figure 1 Schematic of Cartesian and radial grids for area integration over a circular cross-section. Some combinations of incident $\left(L_{1}\right.$, green) and diffracted $\left(L_{2}\right.$, blue) paths lengths (all vectors drawn from grid points to circumference) are indicated for diffraction angles of $2 \theta=90^{\circ}$. 
All possible distances that may occur for any arbitrary grid point $\mathbf{P}$ at any diffraction angle can be deduced from the behaviour of the points (A) along a single line between the origin $(\mathbf{O})$ and the edge (E) of a unit circle, as schematically indicated in Figure 2. For simplicity, these points are taken to lie along the positive $x$-axis of a Cartesian plane whose origin coincides with that of the unit circle, and thus have Cartesian coordinates of $(a, 0)$ where $0 \leq a \leq 1$. All points along this line also have identical radial coordinates of $(a, 0)$, since this line corresponds to a radial angle of $\phi=0$. For each point $\mathbf{A}$ along this line, the distance that any arbitrary ray with an angle $\omega$ (defined relative to the positive $x$-axis) will have to travel to intersect the circumference of the circle at a point $\mathbf{X}$ along the line $\mathbf{A X}$ can be directly calculated using Equation 4.

$$
d(a, \omega)=-a \cos \omega+\sqrt{a^{2} \cos ^{2} \omega+1-a^{2}}
$$

This is the positive root of $d$ obtained using the quadratic formula after substituting the relationships $x$ $=a+d \cos \omega$ and $y=d \sin \omega$ into the equation of a unit circle $\left(x^{2}+y^{2}=1\right)$, and simplifying the result.
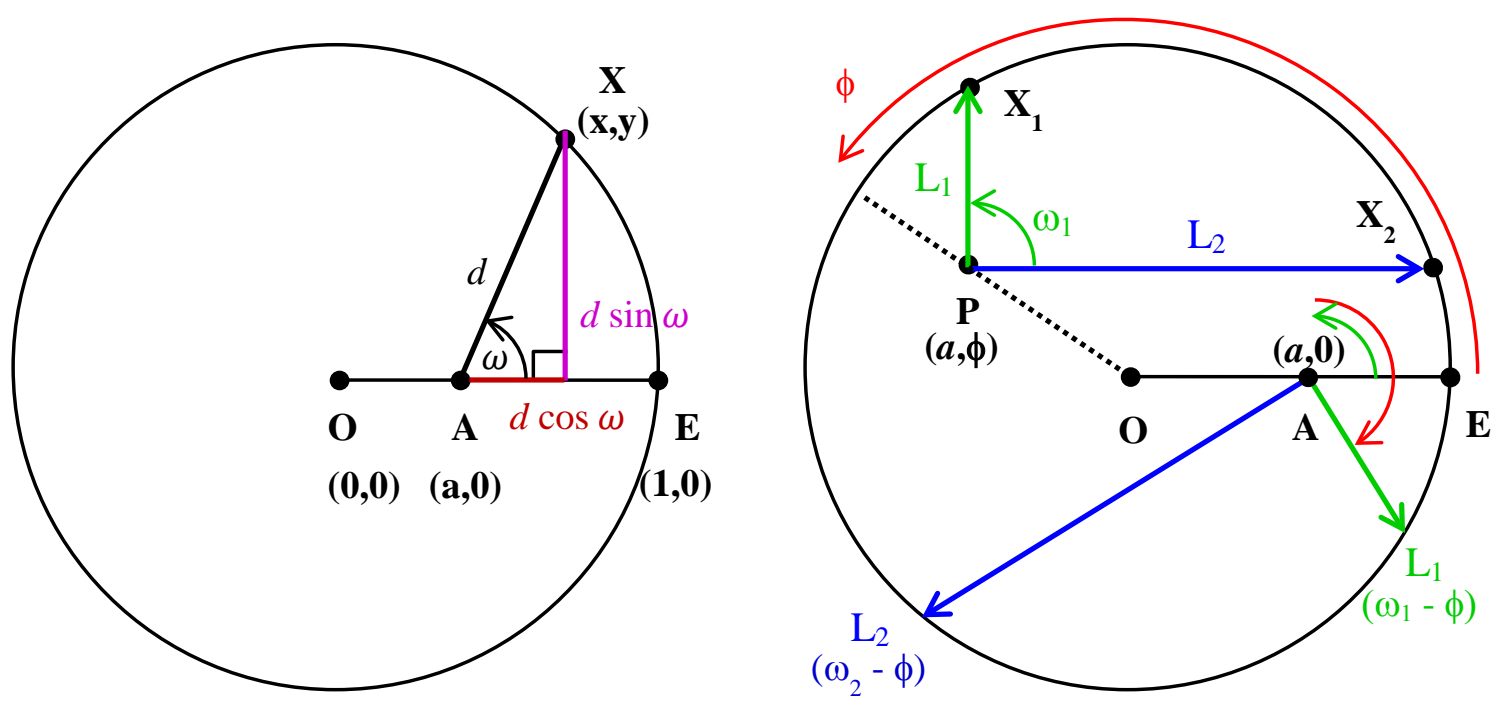

Figure 2 All distance information needed for the numerical calculation of cylindrical absorption corrections can be obtained from a single line of points $\mathbf{O E}$ on a unit circle. The distance from each point $\mathbf{A}$ on this line to the circle circumference (point $\mathbf{X}$ ) in each direction $\omega$ must be calculated. The two distances $L_{1}=\mathbf{P} \mathbf{X}_{1}$ traversed by an incoming X-ray (angle $\omega_{1}$ to positive $x$-axis) and $L_{2}=\mathbf{P} \mathbf{X}_{2}$ traversed by an outgoing X-ray (angle $\omega_{2}$ ) for any arbitrary point $\mathbf{P}$ with radial coordinates $(a, \phi)$ within the circle can always be mapped onto the distance from a point $\mathbf{A}$ on the specific line $\mathbf{O E}$ with radial coordinates $(a, 0)$ in the directions of $\left(\omega_{1}-\phi\right)$ and $\left(\omega_{2}-\phi\right)$, respectively. Note that the angles $\omega_{1}$ and $\omega_{2}$ are calculated based on a common convention (vector from grid point to circumference), making the vector associated with $L_{1}$ aligned opposite to the direction that the incident X-rays travel. 
It is computationally convenient to calculate the distance from point $\mathbf{A}$ to the circle circumference for a $1 \mathrm{D}$ matrix of angles (i.e., $\omega=0,1, \ldots, 359$ ). Both the distance an incoming X-ray (with angle $\omega_{1}$ ) will have to travel to reach point $\mathbf{A}$ as well as the distance that the outgoing $\mathrm{X}$-ray (with angle $\omega_{2}$ ) will have to travel from A to leave the sample simply can be looked up from this matrix of distances, $d(a, \omega)$, giving the two results of $L_{1}=d\left(a, \omega_{1}\right)$ and $L_{2}=d\left(a, \omega_{2}\right)$. Furthermore, any arbitrary point $\mathbf{P}$ within the unit circle with radial coordinates of $(a, \phi)$ can be mapped back onto a point $\mathbf{A}$ on this line with radial coordinates of $(a, 0)$ through rotation by the angle $-\phi$. Thus it is generally true that the distance from any arbitrary point $\mathbf{P}$ to the circle circumference in directions of $\omega_{1}$ and $\omega_{2}$ is exactly equal to the distance from point $\mathbf{A}$ to the circle circumference in directions of ( $\omega_{1}$ $-\phi)$ and $\left(\omega_{2}-\phi\right)$, respectively. This can be written in the form of a mathematical equality between two points with equal radial components of their coordinates $(r=a)$.

$$
d_{\omega}(a, \phi)=d_{(\omega-\phi)}(a, 0)
$$

Here, the general expression $d_{\omega}(r, \phi)$ denotes the distance between a point with radial coordinates of $(r, \phi)$ and the circumference of the unit circle along a ray which makes an angle $\omega$ to the positive $x$ axis. A graphical illustration of this equality is provided in Figure 2. The 2D matrix $(\omega \times a)$ of the distance distribution $d_{\omega}(a, 0)$ for all points on the line OE contains all distance information necessary to calculate transmission factors by area integration over the entire circular cross-section not just for one value of the diffraction angle $\theta$ but for any value of $\theta$, an advantage will be discussed more later.

The use of a dimensionless unit circle to describe the geometry of this problem simplifies the derivation, and can be considered to describe the specific case where $\mu R=1$, with the capillary radius $R$ being equal to half of its diameter. It is computationally trivial to linearly rescale distances obtained in this manner when calculations for other values of $\mu R$ are needed, though the assignment of distances will continue to be described in terms of the unit circle throughout this work in order to provide an intuitive understanding of the underlying mathematics. A polar plot showing the angledependence of the distance to the circumference of the unit circle for different radial coordinates of $a$ along the line $\mathbf{O E}$ is given in Figure 3 together with a conventional Cartesian plot of the same distance distributions. The maximum distance that an $\mathrm{X}$-ray can traverse to reach a point $\mathbf{A}$ is equal to $(1+a)$ in these dimensionless units. For the point at the origin of the circle ( $\mathbf{O}$, red line), the distance traversed will be 1 regardless of the angle $\omega$ along which the X-ray is travelling. For a point at the edge of the unit circle (E, purple line), an incident X-ray can travel up to a length of 2 (for $\omega=0^{\circ}$ ), and the diffracted X-ray can also travel up to a length of 2 (for $\omega=180^{\circ}$ ), though for half of all possible angles $\left(0^{\circ} \leq \omega \leq 90^{\circ} ; 270^{\circ} \leq \omega \leq 360^{\circ}\right)$ the distance from this edge point $\mathbf{E}$ to the circle circumference will take the opposite extreme value of zero. 

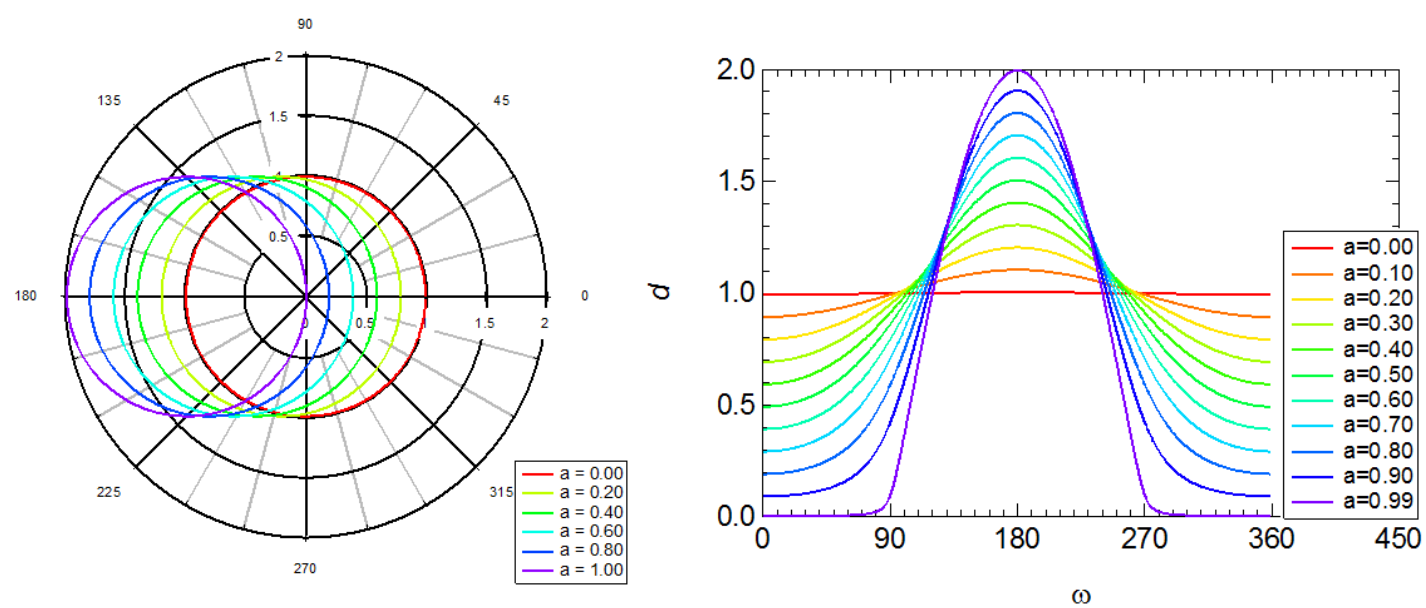

Figure 3 Polar and Cartesian plots showing the distance $d(a, \omega)$ from a point with radial coordinates $(a, 0)$ to the unit circle circumference in all different directions $\omega$ for specific values of $a$. This distance $d$ always falls in the range of $0-2$, but has a much stronger angle dependence for larger values of $a$.

\subsection{Symmetry in incident/diffracted $X$-ray path lengths}

While the calculation of distances $(d)$ is first done in a general manner, the calculation of absorbances requires knowledge of the separate path lengths traversed by the incident $\left(L_{1}\right)$ and diffracted beams $\left(L_{2}\right)$ to each point in the radial grid illustrated in Figure 1. This calculation can be simplified through the recognition that the distribution of path lengths $\left(L_{1}\right)$ traversed by incident Xrays with one common angle $\omega_{1}$ to the ring of grid points at a radius of $a$ from the center of the unit circle with a range of coordinates of $\left(a, 0 \leq \phi<360^{\circ}\right)$ is exactly identical to the distribution of distances for the single point with coordinates of $(a, 0)$ over a range of angles of $0 \leq \omega<360^{\circ}$ that is plotted in Figure 3, and which follows the relationship previously given in Equation 5. Furthermore, the path lengths $\left(L_{2}\right)$ traversed by outgoing X-rays at the specific angle $\omega_{2}$ will have exactly the same orientation-dependent distance distribution as the incident path lengths $\left(L_{1}\right)$ but with a phase shift of $\left|\omega_{2}-\omega_{1}\right|$ (value must be in the range of 0 to $180^{\circ}$ ). The conventional diffraction angles $\theta_{\mathrm{D}}$ and $2 \theta_{\mathrm{D}}$ can be related to this phase shift through Equation 6.

$$
\left|\omega_{2}-\omega_{1}\right|=180^{\circ}-2 \theta_{\mathrm{D}}
$$

This is graphically illustrated for the specific case of a points near the edge of the capillary ( $a=0.90$ ) in Figure 4. The path lengths of the incident beam (red line) and diffracted beam (dashed blue lines) are completely out of phase (shifted by $180^{\circ}$ ) when $2 \theta_{\mathrm{D}}=0^{\circ}$, but become more and more in phase as the diffraction angle increases to $2 \theta_{\mathrm{D}}=180^{\circ}$, an angle for which the distributions must be perfectly in phase since the incident and diffracted X-rays follow identical paths through the sample. This method 
for calculating the distances $L_{1}$ and $L_{2}$ provides a two-fold acceleration relative to the equivalent calculations for a Cartesian grid as it explicitly makes use of the equivalence of their distance distributions. The numerical implementation is carried out by creating a $2 \mathrm{D}(\omega \times a)$ matrix with distances $d_{\omega}(a, 0)$ calculated as a function of radial coordinate $(0 \leq a<1)$ and $\omega$ angle $\left(0 \leq \omega<360^{\circ}\right)$.
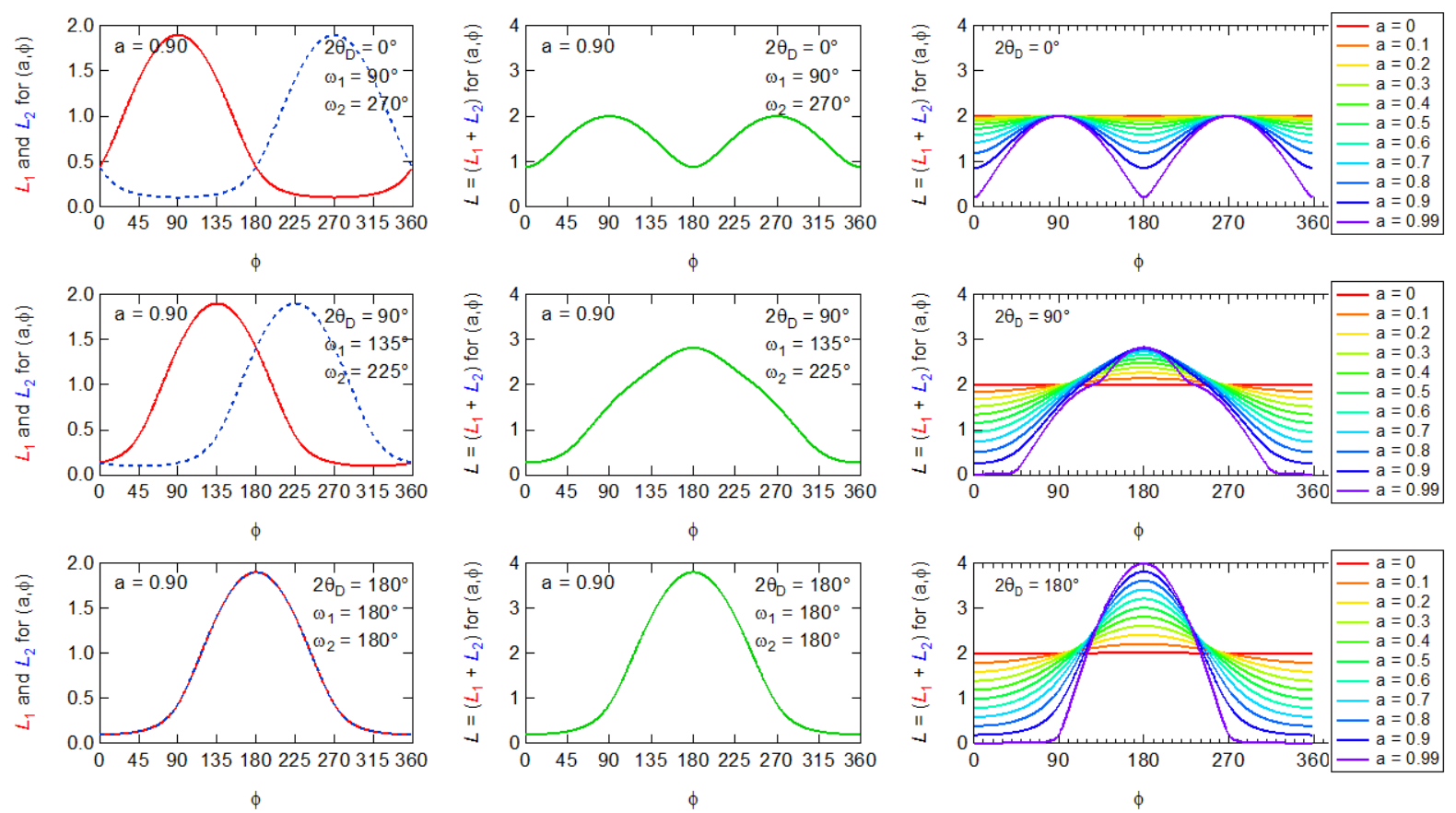

Figure 4 Left: Individual path lengths $\left(L_{1}\right.$ and $\left.L_{2}\right)$ from each point in the ring $(0.90, \phi)$ to the circumference of the unit circle in the incident beam (solid red) and diffracted beam (dashed blue) directions, which are specified by angles of $\omega_{1}$ and $\omega_{2}$, respectively. The phase shift between these two distance distributions is given by $\left|\omega_{2}-\omega_{1}\right|=180-2 \theta_{\mathrm{D}}$, where $\theta_{\mathrm{D}}$ is the conventional diffraction angle. Center: The total distance $L=L_{1}+L_{2}$ travelled by X-rays scattered from each point $(0.90, \phi)$ is shown in green, and is limited to values of $0 \leq d \leq 4$. Right: Distance distributions for additional rings with radial coordinates of $(a, \phi)$ with $0 \leq a \leq 1$ at the same diffraction angles.

Calculation of the overall transmission factor $T_{\theta}$ requires knowledge of the total path length ( $L=L_{1}+L_{2}$ ) traversed by X-rays scattered from each grid element of the cylindrical cross-section. This distance (green line, Figure 4 center) is simply obtained by summing the phase-shifted incident ( $L_{1}$, red) and diffracted beam $\left(L_{2}\right.$, blue) distance distributions for each ring of radial distributions, where the phase-shift is determined by the diffraction angle, $\theta_{\mathrm{D}}$, as specified in Eqn. 6. For the example case of $a=0.90$, the total path length duplicates the single-peaked distribution of the X-ray beams when $2 \theta_{D}=180^{\circ}$, as an identical path through the sample is traversed by the incident and diffracted X-rays. There is still a singly peaked distribution for medium angles diffraction such as $2 \theta_{\mathrm{D}}=90^{\circ}$, which further evolves to a doubly-peaked distribution for low angles such as $2 \theta_{\mathrm{D}}=0^{\circ}$. Condensed plots showing the angular distribution of distances at different radial coordinates $(a=0$, 
$0.1, \ldots 1$ ) for the same diffraction angles of $0^{\circ}, 90^{\circ}$, and $180^{\circ}$ are also provided in Figure 4 (right). The range of possible total distances is limited to values of $0 \leq d \leq 4$ for a unit circle. As expected, the distance distribution at the circle center $(a=0)$ is independent of angle, while a strong angle dependence is found near the edge ( $a \sim 1)$. When these unit circle distances are linearly rescaled by multiplying by $\mu R$ (the product of the linear absorption coefficient and the capillary radius), the total sample transmission factor $T_{\theta}$ can be simply calculated using Equation 3 .

While the present method provides minor advantages in the calculation of the distance distributions for diffraction at a single angle, it presents a major advantage when calculating transmission factors over multiple angles since every different diffraction angle evaluated can utilize the same distance distribution calculated for a generic unit circle. The only diffraction-angle dependent parameter that needs to be changed for different diffraction angles is the angular offset $\left(\omega_{2}\right.$ - $\omega_{1}$ ) between $L_{1}$ and $L_{2}$, and thus for each different angle calculated the only additional work will involve a new summation of $L_{1}$ and $L_{2}$ and the re-evaluation of the area integral in Equation 3 using the grid of distances obtained in this manner. This is illustrated schematically in Figure 5, where the distance distributions for the ring of points at $a=0.90$ were obtained for a variety of diffraction angles $\theta_{\mathrm{D}}$ from $0^{\circ}$ to $180^{\circ}$ without recalculating the distance distributions of $L_{1}$ and $L_{2}$ used for the first angle chosen. The number of distance calculations need to model a diffraction pattern over an $A \times B$ grid for $C$ different diffraction angles and $D$ different values of the absorption coefficient is generically $2 A B C D$, where the factor of 2 indicates the separate calculations need for the incident $\left(L_{1}\right)$ and diffracted $\left(L_{2}\right)$ beam paths. In the present method, the number of distance calculations is reduced to $\mathrm{AB}$, thus reducing the problem by two dimensions if a range of $\mu R$ values are being investigated, by one dimension if transmission factors are desired for a single $\mu R$ value $(D=1)$, and with no reduction in dimensionality if the transmission factor calculation is only for a single diffraction angle and a single $\mu R$ value ( $C=1$ and $D=1)$. Although the calculation of distances can be simplified using the present methods, it is noted that the evaluation of the exponential terms cannot be simplified and must still be done over the full dimensionality of the system. The computer code for carrying out these calculations was written as a macro for the Igor Pro (WaveMetrics) software, and is provided in the Supporting Information. Even without having access to the computational advantages of using a formal compiler and a dedicated runtime environment, calculation of absorption coefficients to high precision could be done at about twenty per second for each combination of $\mu R$ and diffraction angle using code that was optimized for simplicity rather than speed. 


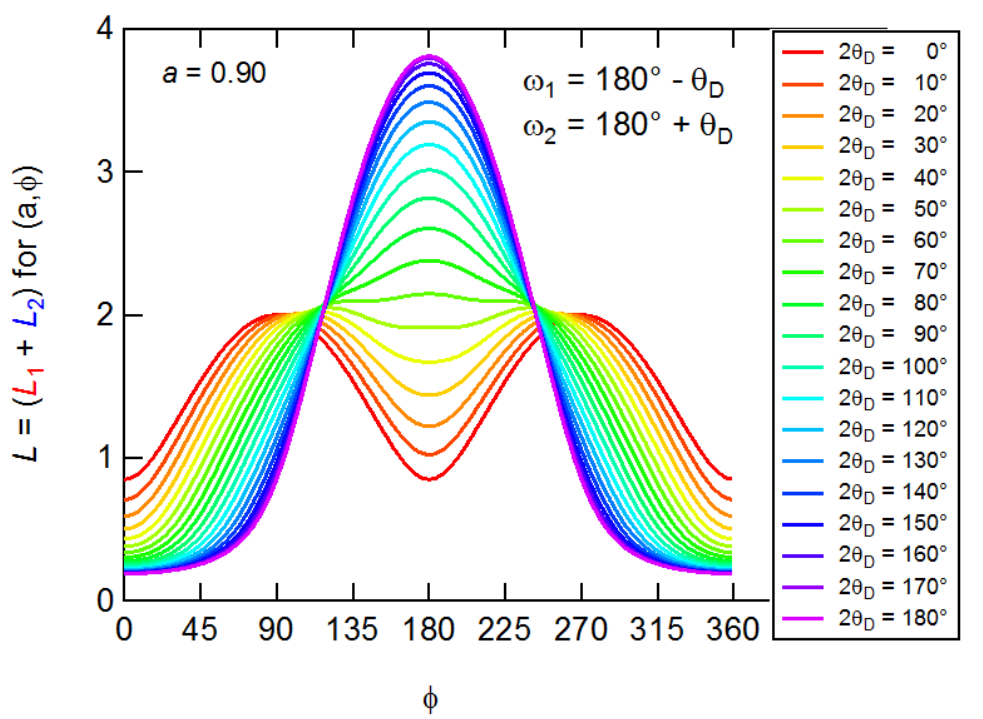

Figure 5 Distribution of total distances $L=L_{1}+L_{2}$ travelled by scattered X-rays from all points with radial coordinates $(a, \phi)$ for many different diffraction angles $\theta_{\mathrm{D}}$ can be obtained after only once carrying out the calculation of the distance distribution $d_{\omega}(a, 0)$, as was shown in Figures 3 and 4 . This same distribution is the repeatedly utilized to calculate $L$ for different diffraction angles by applying variable phase shifts (equal to $\left.2 \theta_{\mathrm{D}}\right)$ to $d_{\omega}(a, 0)$ before summing the distances in this distribution associated with $L_{1}\left[d_{\omega}(a, 0)\right]$ and $L_{2}\left[d_{\omega+2 \theta}(a, 0)\right]$. The specific case of $a=0.90$ is shown in this figure.

\section{Results and Discussion}

\subsection{Transmission factors}

The accuracy of the present algorithm was first verified by comparing calculated values of the transmission factor, $T$, to those calculated analytically by Dwiggins at angles of $2 \theta_{\mathrm{D}}=0^{\circ}$ and $180^{\circ}$, as shown in Table 1. In can be seen that the beam attenuation for data collected in a reflection condition $\left(0^{\circ}\right)$ is always less than for data collected in the straight-through transmission condition $\left(180^{\circ}\right)$, and that this difference becomes more severe as $\mu R$ increases. Grid dimensions of about $400 \times 400$ were typically sufficient to achieve agreement of $0.1 \%$ with the analytical attenuation factors for $\mu \mathrm{R}$ values of 5 or less. The purpose of applying an absorption correction to diffraction data is most typically to correct for the relative changes in peak intensity due to the differential absorption for peaks measured at different diffraction angles. A practical goal might be to numerically calculate the ratio between $T_{\theta}$ at $0^{\circ}$ and $90^{\circ}$ with $1 \%$ accuracy, as the packed density of a powder sample is expected to fluctuate by this amount or more in a typical capillary or can. This level of accuracy in the $T_{0} / T_{90}$ ratio can typically be achieved with reduced grid dimensions of $50 \times 50$ over the same range of $\mu R$. The graphical and numerical results in this work were calculated using a $360 \times 360$ radial grid unless otherwise specified. Complete results for the calculation of transmission factors are provided in a text 
data file in the Supporting Information for values of $0 \leq \mu R \leq 2$ (increments of 0.01 ) and for $0 \leq \mu \mathrm{R} \leq$ 20 (increments of 0.1 ) over angles of $0^{\circ} \leq \theta_{\mathrm{D}} \leq 90^{\circ}$ (steps of $5^{\circ}$ ).

Table 1 Values of $\mathrm{A}_{\theta} *=1 / \mathrm{T}_{\theta}$.

\begin{tabular}{lllll}
\hline & Analytical & Present & Analytical & Present \\
$\mu \mathrm{R}$ & $\theta_{\mathrm{D}}=0^{\circ}$ & $\theta_{\mathrm{D}}=0^{\circ}$ & $\theta_{\mathrm{D}}=90^{\circ}$ & $\theta_{\mathrm{D}}=90^{\circ}$ \\
0 & 1 & 1 & 1 & 1 \\
0.5 & 2.300 & 2.299 & 2.050 & 2.049 \\
1.0 & 5.091 & 5.089 & 3.389 & 3.387 \\
1.5 & 10.74 & 4.863 & 4.859 \\
2.0 & 21.44 & 21.42 & 6.389 & 6.383 \\
2.5 & 40.10 & 40.07 & 7.936 & 7.928 \\
3.0 & 70.12 & 70.07 & 9.492 & 9.482 \\
5.0 & 363.0 & 363.4 & 15.75 & 15.73 \\
10 & & 3,162 & & 31.41 \\
20 & & 29,890 & & 62.90 \\
\hline
\end{tabular}

Analytical results are values from Dwiggins, 1972.

The transmission factors, $T_{\theta}$, calculated for some selected values of $\mu R$ between 0 and 20 are plotted on a linear scale in Figure 6, and these are shown together with the corresponding attenuation factors $A_{\theta}{ }^{*}$ which are shown on a logarithmic scale. It can be seen that both the magnitude and the angle dependence of the overall sample absorbance increases with increasing $\mu R$. The sample $\mu R$ must be below $\sim 1.5$ in order to for the incident beam intensity to not be reduced by more than 10 -fold at low angles, and below $\sim 3.5$ to avoid a 100 -fold reduction. Since peak/noise ratios are typically less than 100 in most diffractometers, this degree of attenuation due to absorption can have a strongly deleterious effect on the quality of measured diffraction data. However, the attenuation factor must not be considered in isolation when designing the optimum sample configuration for a capillary diffraction experiment, as will be discussed in more detail later. 

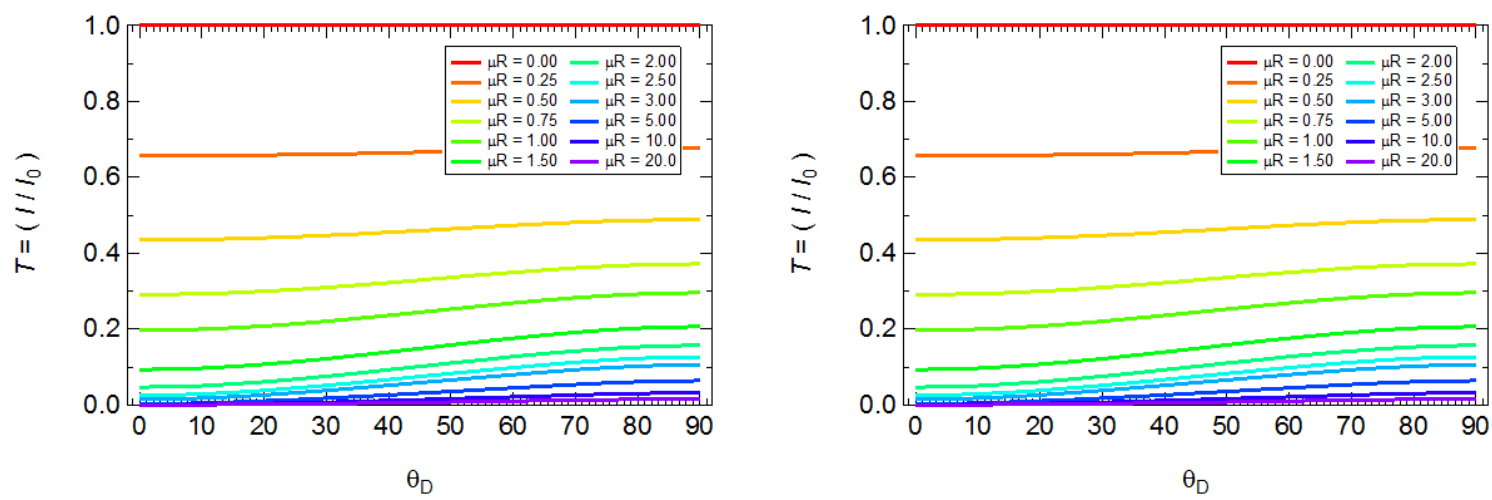

Figure 6 (a) Angle-dependence of transmission factors ( $T$ ) for capillaries with different values of $\mu R$. (b) Angle-dependence of attenuation factor $A^{*}=1 / T$ for capillaries with different values of $\mu R$ plotted on a logarithmic scale, allowing the responses of highly absorbing samples to be resolved.

In addition to examining the absolute transmission factors, it is also important to consider the relative transmission factors as it is the angle-dependence of the absorption that causes the wellknown problems with the determination of displacement parameters during structural refinements. At higher diffraction angles, the displacement of atoms from their average positions results in a larger reduction in the coherent scattering than for lower angles, causing the intensity of high angle peaks to be on average reduced relative to the low angle peaks. Absorption effects have the opposite angular dependence, preferentially suppressing the intensity of low angle peaks. This is perhaps easiest to see when the transmission factors for a given value of $\mu \mathrm{R}$ are normalized to their maximum value (Figure 7), which occurs at $\theta_{\mathrm{D}}=90^{\circ}$. This intensity at low angles is reduced relative to that at $\theta_{\mathrm{D}}=90^{\circ}$ by about $10 \%$ for $\mu \mathrm{R}=0.5$, about $50 \%$ for $\mu \mathrm{R}=1.5$, and more than $90 \%$ for $\mu \mathrm{R}=5$.

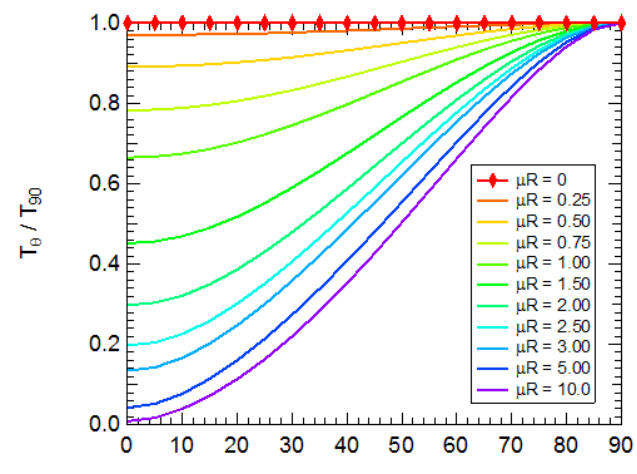

(a)
$\theta_{\mathrm{D}}$

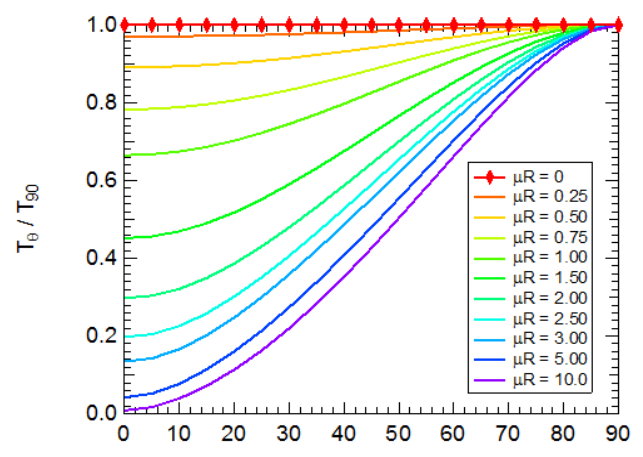

(b)
$\theta_{\mathrm{D}}$

Figure 7 Normalized transmission factors (relative to $T_{90}$ ) for different $\mu R$ values plotted (a) as a function of angle and (b) as a function of $\sin ^{2} \theta_{D}=\lambda^{2} / d^{2}$, highlighting their nearly linear dependence of $T / T_{90}$ on $\sin ^{2} \theta_{\mathrm{D}}$ (behaviour also exhibited by displacement parameters, Eqn. 7). 
When structural refinements neglect to correct for absorption effects, the refined values of displacement parameters will be reduced relative to their true values, and will even refine to aphysical negative values when very strong absorption effects are present. Isotropic displacement parameters $(B)$ depend on the diffraction angle $\left(\theta_{\mathrm{D}}\right)$ and radiation wavelength $(\lambda)$ according to Equation 7.

$$
f=f_{0} e^{-B \sin ^{2} \theta_{D} / \lambda^{2}}
$$

It has been previously noted that neglecting to correct for modest absorption effects will lead to nearly linear offsets in the values of displacement parameters obtained from the refinement of powder diffraction data (Hewat, 1979). This is reflected in the nearly linear behaviour observed when normalized transmission factors are plotted against $\sin ^{2} \theta_{\mathrm{D}}$ (Figure 7). Such behaviour also justifies the fitting of angular dependence of transmission factors to an $N$ term expansion of $T_{\theta}=$ $\sum_{i=0}^{N} L_{i} \sin ^{2 i} \theta_{D}$, as has been previously proposed (Dwiggins, 1975). When this was attempted for the present data, it was observed that a single term fit works well for values of $\mu R<1$, three terms work well up to $\mu \mathrm{R}<5$, and more terms are needed to reasonably model the angle dependence of $\mathrm{T}$ for larger values of $\mu \mathrm{R}$. It is generally the most challenging to fit this angular dependence at the highest and lowest angles, though in practice these regions are the least likely to have peaks of interest in most diffraction experiments. In the absence of severe absorption effects, it is very reasonable to model transmission factors as having a linear dependence on $\sin ^{2} \theta_{\mathrm{D}}$, or equivalently on $1 / d^{2}$ when the Bragg's law relationship between $d$-spacing and angle is considered.

\subsection{Optimum capillary radius}

\subsubsection{Radius dependence}

Using the transmission factors that have been numerically calculated, the optimum capillary radius for a given experiment can be determined. As the capillary radius $R$ is increased, the transmission factor $T$ will always decrease, but the volume of the sample will also increase proportionally to the sample cross-sectional area of $\pi R^{2}$ so long as the sample can fully fit within the beam. The total diffracted beam intensity will therefore scale as $R^{2} T$, though it should be noted that $T$ will depend on both the $\mu R$ product and the diffraction angle, $\theta_{\mathrm{D}}$. It is chosen to denote these dependences as $T_{\theta}(\mu R)$ in the present treatment, where both the subscript and the parenthesis indicate variables on which the function $T$ depends. The relationship for the total diffracted beam intensity, I, can therefore be written as follows:

$$
\begin{aligned}
& \mathrm{I} \propto R^{2} T_{\theta}(\mu R)=\left(1 / \mu^{2}\right)(\mu R)^{2} T_{\theta}(\mu R) \\
& \mu^{2} \mathrm{I} \propto(\mu R)^{2} T_{\theta}(\mu R)
\end{aligned}
$$


A plot of the function $\mu^{2}$ I for different values of $\theta_{D}$ is given in Figure 8, with this function arbitrarily multiplied by $\pi$ to give convenient values since it was noted that $T_{90}(\mu \mathrm{R})=1 /(\pi \mu \mathrm{R})$ for large values of $\mu \mathrm{R}$. This presumably reflects a simplification of the $2 \mathrm{D}$ cylindrical absorbance calculation to a unidirectional problem for the special case of $2 \theta_{D}=180^{\circ}$ in which the cylindrical sample behaves like a thin plate (where $A^{*}$ scales as $1 / 2 \mu$ ) or very thin annulus (i.e. curved plate) due to the limited penetration of radiation when $\mu R$ is large, though the author is not aware of an existing geometrical proof of this proposed relationship. It therefore follows that the diffracted intensity will increase linearly with $R$ for $\theta_{\mathrm{D}}=90^{\circ}$. A larger capillary radius is always desirable for maximizing the intensity of diffraction peaks at $\theta_{\mathrm{D}}=90^{\circ}$, a trend which persists for angles as low as $\theta_{\mathrm{D}} \sim 25^{\circ}$. This is particularly relevant for experiments which aim to maximize the scattering of peaks with a low $d$ spacing (which typically occur at high angles) and thereby the total information content for structural refinement, in analogy to the use of variable counting times to enable longer collection times at higher angles (Madsen \& Hill, 1994). At angles below $\theta_{\mathrm{D}} \sim 25^{\circ}$, a maximum in the diffraction intensity will occur for $\mu R \sim 1.4$ as has been noted previously (Schmitt \& Ouladdiaf, 1998), and the drop off in intensity beyond this maximum is more rapid for lower diffraction angles. For the limiting case of $\theta_{\mathrm{D}}$ $=0^{\circ}$, intensities within $\sim 80 \%$ of the peak value will be obtained for $0.8<\mu R<2.2$.
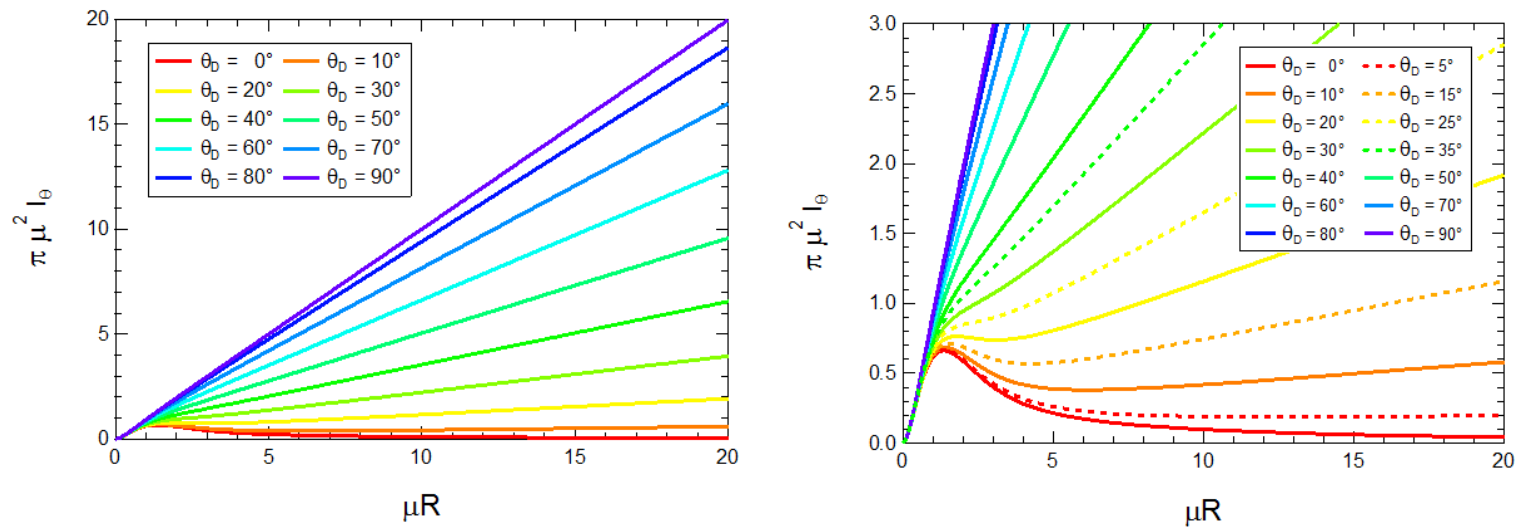

Figure 8 Dependence of relative diffraction peak intensity on $\mu$ and $R$ for different diffraction angles, $\theta_{\mathrm{D}}$, where $\mu^{2} \mathrm{I}=(\mu R)^{2} T_{\theta}(\mu R)$. For a constant value of $\mu$, the net intensity may decrease for larger values of $\mu \mathrm{R}$ when $\theta_{\mathrm{D}}<25^{\circ}$, but will always increase with increasing $R$ for higher diffraction angles. An expanded view of the behaviour at low values of $\mu R$ is provided in Figure $\mathrm{S} 1$.

The results of the present analysis substantially differ from the simpler conventional model of assuming that the absorption of a single point at the center of the capillary is representative of the entire sample (leading to $T=\mathrm{e}^{-2 \mu \mathrm{R}}$ and $\mathrm{I} \propto R^{2} \mathrm{e}^{-2 \mu \mathrm{R}}$ ). This single-point approximation completely misses the strong angular dependence of the diffraction intensity caused by absorption effects and predicts a significantly different intensity maximum at $\mu R=1$, as is shown in Fig. S1. While the 
present treatment allows the dependence of diffracted beam intensity on $\mu R$ to be predicted in a very universal manner which depends only on the product of $\mu$ and $R$, it should be noted that the prefactor of the vertical axis contains a factor of $\mu^{2}$ which will depends on $\mu$ alone and which will clearly impact the quality of experimental data. For two samples with $\mu R=1$, a sample with $\mu=10$ and $R=$ 0.1 will give diffraction peaks that are 25 times weaker than those for a sample with $\mu=2$ and $R=$ 0.5 , assuming all else is equal. This simple calculation highlights the important conclusion that knowledge of the $\mu R$ product alone is not enough for making good predictions about the optimal sample loading for diffraction experiments.

It is emphasized that the present results apply for the ideal diffraction geometry only, and neglect potential experimental issues such as an inhomogeneous beam intensity distributions, inhomogeneous sample packing, and absorption by the cylindrical capillary or can. Furthermore, the best data will be collected when the signal/noise ratio is maximized, and the present treatment implicitly assumes that the background noise will scale linearly with the sample volume. For samples that fluoresce, this is likely a poor assumption. It should be noted that the angle-dependence of the transmission factor is always less for lower values of $\mu R$, and as a consequence, structural models will be more robust against imperfections in the modelled absorption when samples with smaller $\mu R$ values are used. This potentially enables a more accurate determination of the structural model using data sets which have a worse overall signal/noise ratio relative to samples with larger $\mu R$ products, and is especially advantageous when the determination of accurate absolute values of displacement parameters is the experimental goal. While the present treatment provides a rational theoretical basis for designing diffraction experiments, both beamline-specific and sample-specific experimental validation of these predictions are still recommended.

\subsubsection{Density dependence}

The overall intensity of diffraction can alternatively be optimized by modifying the density of the sample, a goal which is typically accomplished by carrying out sample dilution with a material that has a negligible absorbance. The choice of diluent may vary depending on the nature of the diffraction experiment, but might involve materials such as powdered glass, cork, solid or hollow polymeric microspheres, aerogels, or amorphous boron. We define a density reduction factor, $F$, which describes the reduction in sample density $(\rho)$ relative to the original value prior to modification ( $\rho_{0}$ ) through the relationship $\rho=\rho_{0} / F$, where values of $1<F<\infty$ correspond to physically plausible dilution conditions, and values of $0<F<1$ correspond to an increased sample density achieved by compaction. If a constant value of $\mu R$ is maintained throughout dilution, the capillary radius will need to increase from $R$ to $F R$ to achieve this. As a result, the capillary volume will increase by a factor of $F^{2}$ while the amount of material in the beam per unit volume will decrease by a factor of $F$ relative to a sample of the same diameter which was not diluted, as expressed below in Equation 10. 


$$
\begin{aligned}
& \mathrm{I} \propto(1 / F)(\mathrm{FR})^{2} T_{\theta}(\mu R) \\
& \mathrm{I}_{\mathrm{F}} / \mathrm{I}_{1}=F
\end{aligned}
$$

Thus the intensity of the diffracted beam will vary linearly with $\mathrm{F}$ in the absence of other volumedependent effects when dilution is carried out in a manner that preserves $\mu R$ (Eqn. 11), and dilution will thus always increase the measured diffraction intensity when the capillary radius can be increased in a proportional manner.

It is of course also possible to carry out dilution while allowing the overall $\mu R$ value for the sample to change. For the dilution of a given sample, both the argument of the transmission function $T_{\theta}$ and the amount of diffracting sample will be rescaled by a factor $F$, leading to Equations 12 and 13 .

$$
\begin{aligned}
& \mathrm{I} \propto(1 / F) T_{\theta}(\mu R / F) \\
& (\mu R) I \propto(\mu R / F) T_{\theta}(\mu R / F)
\end{aligned}
$$

In this approach, it makes sense to discuss the transmission factor as a function of $\mu \mathrm{R} / \mathrm{F}$ rather than as a function of $\mu R$ (as was done when examining the radius dependence of diffraction intensity). A plot of the function $(\mu R) \mathrm{I}$ for different values of $\theta_{\mathrm{D}}$ is given in Figure 9, with this function again arbitrarily multiplied by $\pi$ to give convenient values. It should be noted that unlike the previous transformation (Eqn. 8), a factor of $R$ remains on the left side of Eqn. 13 and this must be explicitly considered when determining the most appropriate dilution factor, in contrast to the prefactor of $\mu$ which is constant for a given material. For a constant capillary radius (fixed values of $\mu$ and $R$ ), the dilution of samples with values of $\mu R$ larger than the peak value of these graphs (which occur for $0.6<\mu R / F<0.9$ for diffraction angles of $0-50^{\circ}$ ) should lead to an increase in the diffracted beam intensity. It should be noted that concentrating samples with values of $\mu R / F$ less than the peak value (for example, by increasing their packing density if a density less than the theoretical one is taken to be the reference density, $\rho_{0}$ ) leads to values of $F<1$ and will enhance the measured diffraction intensity. It should be noted that the diffraction intensity at $90^{\circ}$ can never be increased by dilution since $\mathrm{T}_{90}(x)=1 /(\pi x)$ for large values of $x$. Therefore, $\mathrm{T}_{90}(\mu R / F)$ will never fall off quickly enough with increasing $F$ to exceed for the $1 / F$ loss of intensity resulting from the reduced amount of sample in the beam. This weaker $x$ dependence of the transmission factor $T_{\theta}(x)$ at high angles is responsible for the peak maximum shifting to higher values of $\mu \mathrm{R} / \mathrm{F}$ for large diffraction angles $\theta$ in Figure 9 . 

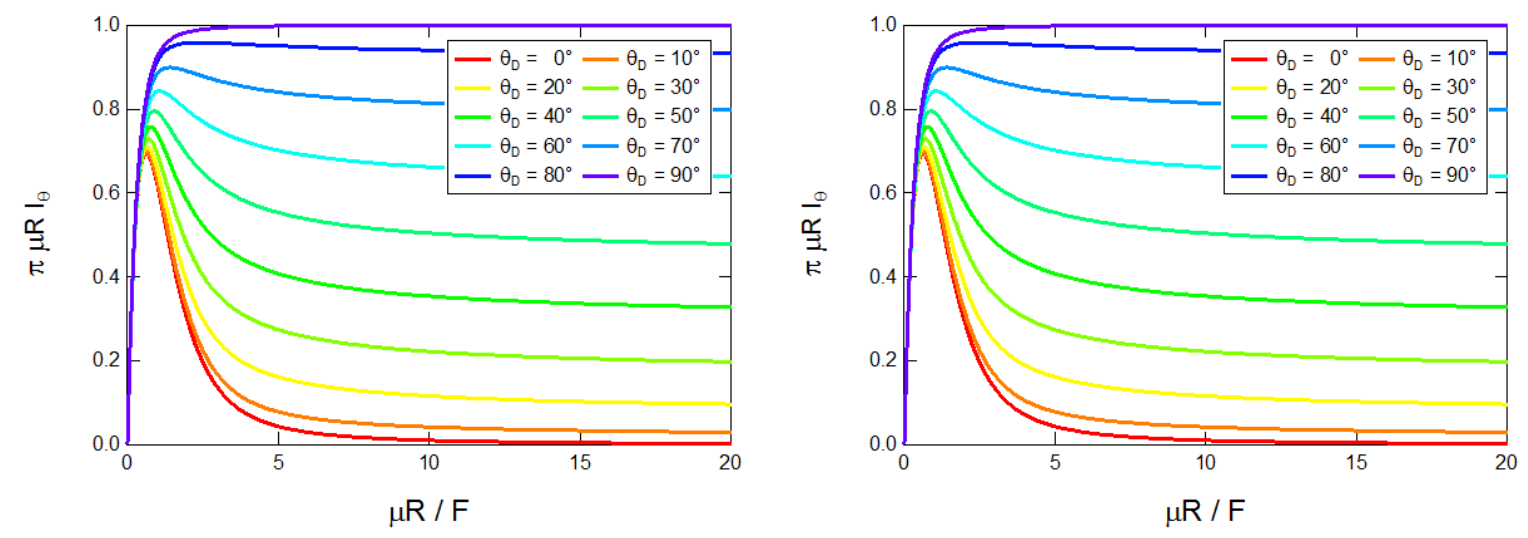

Figure 9 Effect of dilution on relative diffraction peak intensity, where $\mu R I_{\theta}=(\mu R / F) T_{\theta}(\mu R / F)$ for different diffraction angles, $\theta_{\mathrm{D}}$. The maximum intensity will generally occur when $\mu R / F \sim 0.75$, where the factor $F$ represents a reduction in sample density such that $\rho=\rho_{0} / F$.

Based on this analysis, the optimization of the diffracted beam intensity can be achieved by first choosing the maximum diameter capillary compatible with the incident beam, since Eqns. 10 and 11 suggests that a smaller diameter capillary will never be optimal for the best value of the $\mu R$ product. Next, Figure 9 can be used to determine the optimal density reduction factor $F$ for the angles of interest, which will typically occur for $\mu R / F \sim 0.75$. The data in Figure 9 are also provided electronically as Supporting Information. Thus the optimal value of $F$ can be estimated by Equation 14 for a given capillary radius, $R$, and a linear absorption coefficient $\mu$ for the material of interest.

$$
F_{\text {optimal }} \sim(4 / 3) \mu R
$$

\subsubsection{Sample calculation}

An example of this procedure is given for the perovskite compound $\mathrm{PbZrO}_{3}$, which is a strongly absorbing material due to the presence of both $\mathrm{Pb}(\mathrm{Z}=82)$ and $\mathrm{Zr}(\mathrm{Z}=40)$. The room temperature density of this compound is $8.1 \mathrm{~g} / \mathrm{cm}^{3}$, and the linear absorption coefficient of this compound at the typical wavelength of the 11 -BM synchrotron beamline at the APS $(\lambda=0.41 \AA)$ is $\mu$ $=18.6 \mathrm{~mm}^{-1}$. For a capillary with a radius of $R=0.40 \mathrm{~mm}$, it is calculated that $\mu R=7.44$. When multiplied by $4 / 3$, the dimensionless optimal dilution factor is predicted to be $F=9.9$. The optimal scattering will be achieved when the volumetric density of $\mathrm{PbZrO}_{3}$ in the capillary is reduced to about $10 \%$ of that of a fully dense single crystal. If the non-adsorbing diluent powder packs to $~ 50 \%$ of its theoretical density, then a 1:4 dilution by volume would be appropriate. The expected improvements in intensity are estimated to be $36 \mathrm{x}$ at $2 \theta_{\mathrm{D}}=0^{\circ}, 13 \mathrm{x}$ at $20^{\circ}, 5.3 \mathrm{x}$ at $40^{\circ}, 3.0 \mathrm{x}$ at $60^{\circ}, 1.2 \mathrm{x}$ at $120^{\circ}$, and peaks will be reduced in intensity to $0.89 \mathrm{x}$ of the undiluted value at $2 \theta_{\mathrm{D}}=180^{\circ}$. If the same $\mu R / F$ factor of 0.75 is achieved without adding a non-absorbing diluent by packing $\mathrm{PbZrO}_{3}$ into an $R=0.08$ 
mm capillary with a $50 \%$ packing efficiency, the diffraction intensity would be expected to be $5 \mathrm{x}$ lower than achieved by optimal dilution in an $0.4 \mathrm{~mm}$ capillary. It is also noted that the transmittance factor estimated by the simplistic point model of $\mathrm{e}^{-2 \mu \mathrm{R}}$ is $6 \times 10^{-4}$, which is not far from the numerically integrated values of $T_{0}=8 \times 10^{-4}$, but substantially differs from $T_{10}=22 \times 10^{-4}, T_{30}=100 \times 10^{-4}$, and $T_{90}=430 \times 10^{-4}$, reflecting the fact that most of the diffracted intensity from this strongly absorbing sample is coming from the perimeter and not the center of the capillary at higher diffraction angles.

A sample prepared with $\mu R / F=0.75$ should generally give the highest achievable diffraction peak intensities so long as the assumptions made in these derivations are valid. There are of course many good experimental reasons for choosing not to follow this suggested optimization procedure (choice of a smaller capillary to reduce diffraction peak widths when a diffracted beam monochromator is not used in a parallel beam experiment; choice not to use a diluent when the sample must be fully recovered after the experiment; limited sample availability making it impossible to fill a large diameter capillary; strong sample fluorescence or extinction invalidating the derivation assumptions). In these cases, other figures in this paper will still provide useful guidance in choosing a sample loading procedure that is likely to give the highest quality diffraction data under a different set of experimental constraints. We also note that this method can be easily adapted to calculations of absorption coefficients for out-of-plane scattering or for annular sample shapes, and will present those results in another manuscript.

Acknowledgements This work has been funded by the National Science Foundation under award DMR-0955646.

\section{References}

Bowden, M. \& Ryan, M. (2010). Journal of Applied Crystallography 43, 693-698.

Dwiggins, C. W. (1972). Acta Crystallographica Section A-Crystal Physics Diffraction Theoretical and General Crystallography A 28, 219-220.

Dwiggins, C. W. (1975). Acta Crystallographica Section A A 31, 146-148.

Hewat, A. W. (1979). Acta Crystallographica Section A 35, 248-248.

Huq, A., Hodges, J. P., Gourdon, O. \& Heroux, L. (2011). Zeitschrift für Kristallographie Proceedings 1, 127-135.

Lobanov, N. N. \& alte da Veiga, L. (1998). 6th European Powder Diffraction Conference, Abstract P12-16.

Madsen, I. C. \& Hill, R. J. (1994). Journal of Applied Crystallography 27, 385-392.

Rouse, K. D., Cooper, M. J., York, E. J. \& Chakera, A. (1970). Acta Crystallographica Section A-Crystal Physics Diffraction Theoretical and General Crystallography A 26, 682-691.

Sabine, T. M., Hunter, B. A., Sabine, W. R. \& Ball, C. J. (1998). Journal of Applied Crystallography 31, 47-51.

Schmitt, D. \& Ouladdiaf, B. (1998). Journal of Applied Crystallography 31, 620-624.

Wang, J., Toby, B. H., Lee, P. L., Ribaud, L., Antao, S. M., Kurtz, C., Ramanathan, M., Von Dreele, R. B. \& Beno, M. A. (2008). Review of Scientific Instruments 79. 


\section{Supporting information}

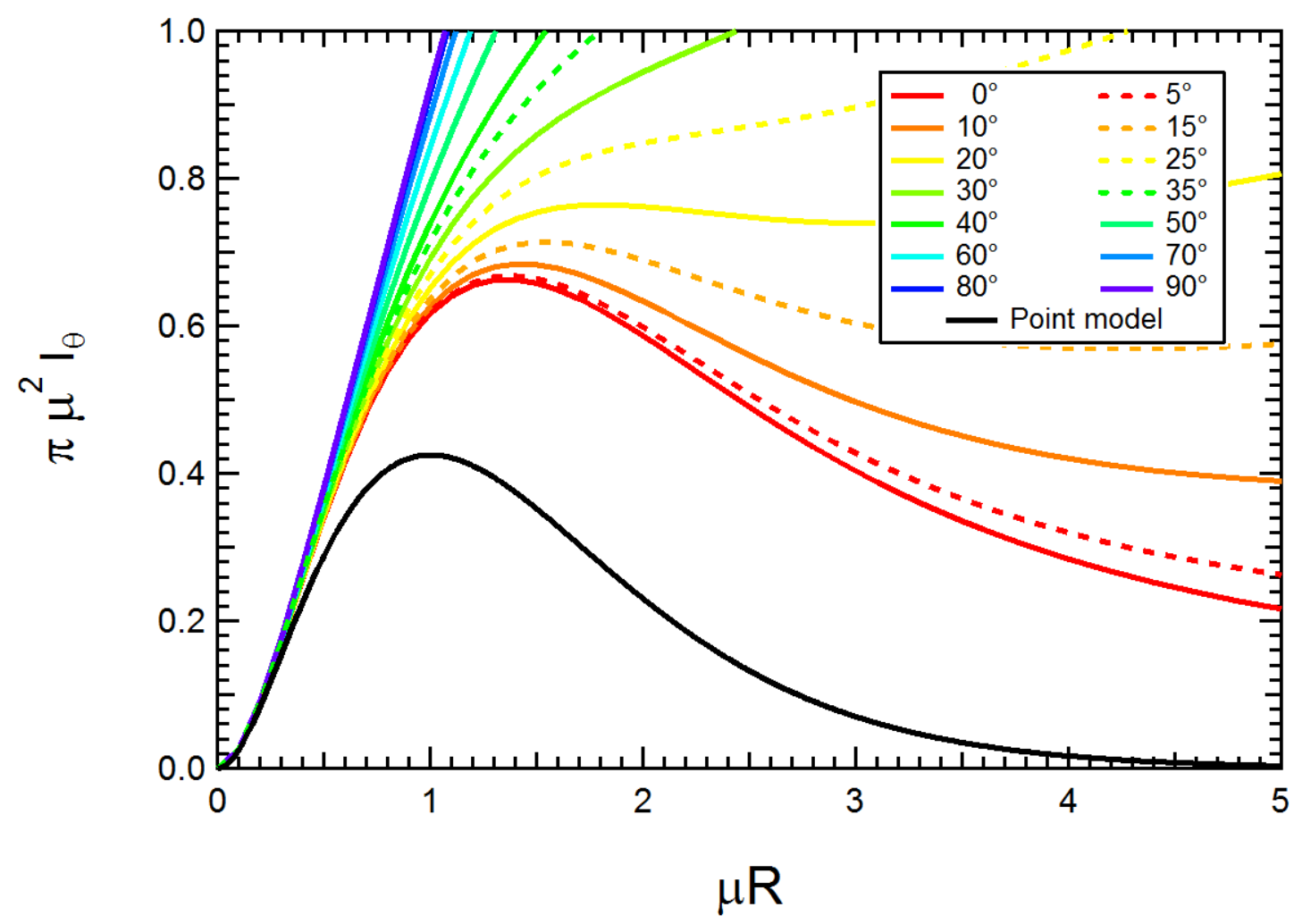

Figure S1 Dependence of relative intensity of diffraction $\pi(\mu R)^{2} T_{\theta}(\mu R)=\pi \mu^{2}$ I compared at different diffraction angles of $0^{\circ} \leq \theta_{\mathrm{D}} \leq 90^{\circ}$ against the estimate made by only considering absorption for a point at the center of the capillary (black line). 